\title{
Stereoselective Synthesis of Chiral 3-Aryl-1-Alkynes from Bromoallenes and Heterocuprates.
}

\author{
Anna Maria Caporusso, ${ }^{* \S}$ Alessia Zampieri, ${ }^{\S}$ Laura Antonella Aronica, ${ }^{\S}$ Donatella Banti ${ }^{\dagger}$ \\ ${ }^{\S}$ Dipartimento di Chimica e Chimica Industriale, Università degli Studi di Pisa, Via Risorgimento 35, \\ 56126 Pisa, Italy; ${ }^{\dagger}$ School of Pharmaceutical and Chemical Sciences, Kingston University, Penrhyn \\ Road, Kingston upon Thames - Surrey, KT1 2EE, United Kingdom
}

capored@dcci.unipi.it

\section{Supporting Information}

\section{Contents:}

General Remarks

${ }^{1} \mathrm{H}-\mathrm{NMR},{ }^{13} \mathrm{C}-\mathrm{NMR}$, GC-MS (\%), IR, Anal. Calcd and Found of compounds 3ad-3ak S3-S4

${ }^{1} \mathrm{H}-\mathrm{NMR},{ }^{13} \mathrm{C}-\mathrm{NMR}$, GC-MS (\%), IR, Anal. Calcd and Found of compounds 3be-3bj $\quad$ S4-S5

${ }^{1} \mathrm{H}-\mathrm{NMR},{ }^{13} \mathrm{C}-\mathrm{NMR}$, GC-MS (\%), IR, Anal. Calcd and Found of compounds 3ce-3cj $\quad$ S6

${ }^{1} \mathrm{H}-\mathrm{NMR},{ }^{13} \mathrm{C}-\mathrm{NMR}, \mathrm{GC}-\mathrm{MS}(\%)$, IR, Anal. Calcd and Found of compounds 3db-3dk $\quad$ S6-S8

${ }^{1} \mathrm{H}-\mathrm{NMR},{ }^{13} \mathrm{C}-\mathrm{NMR}, \mathrm{GC}-\mathrm{MS}(\%)$, IR, Anal. Calcd and Found of compounds 4ad-4ak $\quad$ S8-S9

${ }^{1} \mathrm{H}-\mathrm{NMR},{ }^{13} \mathrm{C}-\mathrm{NMR}, \mathrm{GC}-\mathrm{MS}(\%), \mathrm{IR}$, Anal. Calcd and Found of compounds 4be-4bj $\quad$ S9-S10

${ }^{1} \mathrm{H}-\mathrm{NMR},{ }^{13} \mathrm{C}-\mathrm{NMR}$, GC-MS (\%), IR, Anal. Calcd and Found of compounds 4ce-4cj $\quad$ S10-S11 
General remarks. Infrared absorption spectra (FTIR) were recorded as neat films, and only noteworthy absorptions $\left(\mathrm{cm}^{-1}\right)$ are listed. NMR spectra were recorded in $\mathrm{CDCl}_{3}$ solution at 300 or 200 $\mathrm{MHz}\left({ }^{1} \mathrm{H}\right)$ and $50.3 \mathrm{MHz}\left({ }^{13} \mathrm{C}\right)$. Chemical shifts were determined relative to internal $\mathrm{Si}\left(\mathrm{CH}_{3}\right)_{4}(\delta=0$ ppm) and coupling constants $J$ are in Hz. GC analyses were performed on a chromatograph equipped with a flame ionisation detector (FID), using a $\mathrm{SiO}_{2}$ "Wide Bore" column (DB1, 30m $\times 0.53 \mathrm{~mm}$, $5 \mu \mathrm{m})$ and helium as carrier gas. Preparative GC were performed with SE-30 and Carbowax 20M as stationary phases $(3 \mathrm{~m} \times 0.8 \mathrm{~cm}$ columns $)$. Optical rotations were measured with an automatic polarimeter, using standard cuvettes $(l=0.1$ and $1 \mathrm{dm})$. Analytical thin-layer chromatography (TLC) was performed on aluminium sheets precoated $(0.25 \mathrm{~mm})$ with silica gel and preparative column chromatography on Fluka silica gel 60 (230-400 mesh). Microanalyses were acconplished at the Faculty of Pharmacy, Pisa University.

All air- or moisture-sensitive reactions were carried out in flame-dried glassware under a dry argon or nitrogen atmosphere. Solvents were reagent-grade materials, purified by standard methods, redistilled and stored under argon. The racemic 1-bromo-1,2-dienes, 1a-e, were made and purified as previously described (70-85\% yield $)^{1}$ from the appropriate propargylic alcohols. (S)-1-bromo-1,2-dienes, (S)-1ad, were synthesized from the optically active (R)-carbinols ${ }^{2}$ by reacting the corresponding methanesulfonate esters with $\mathrm{LiCuBr}_{2}$ or $\mathrm{Li}_{2} \mathrm{CuBr}_{3}{ }^{1 \mathrm{c}, \mathrm{d}}$ The enantiomeric purities of compounds (S)1a-d (Table 2) were determined by GC analyses on a Cydex-B chiral column $[25 \mathrm{~m} \times 0.33 \mathrm{~mm}$, $0.25 \mu \mathrm{m}$ heptakis(2,3,6-tri-O-methyl)- $\beta$-cyclodextrin as stationary phase] and/or ${ }^{1} \mathrm{H}$ NMR in $\mathrm{CD}_{3} \mathrm{OD}$ as solvent and heptakis(2,3,6-tri-O-methyl)- $\beta$-cyclodextrin as chiral solvating agent. ${ }^{2 e, 3}$ The arylbromocuprates 2 were prepared by adding, at $0^{\circ} \mathrm{C}, 1$ molar equivalent of $\mathrm{ArMgBr}$ to a well stirred tetrahydrofuran (THF) solution of $\mathrm{LiCuBr}_{2}$, made from stoicheiometric amounts of cuprous bromide 
and lithium bromide; stirring was continued at $0^{\circ} \mathrm{C}$ for $30 \mathrm{~min}$, and the mixture then used immediately. The aryl Grignard reagents $\mathrm{ArMgBr}$ were prepared in THF from the corresponding arylbromides, generally purchased from Fluka, and standardized by titration methods. 4-Bromo-iso-butylbenzene was obtained by a published procedure ${ }^{4}$ starting from commercially available iso-butylbenzene. Zinc cyanocuprates 5 were prepared by stirring, at $-10^{\circ} \mathrm{C}$, the alkylzinc-chlorides $\mathrm{RZnCl}$ with a stoichiometric amount of a THF solution of $\mathrm{CuCN} 2 \mathrm{LiCl}^{5}{ }^{5}$ stirring was continued at $0^{\circ} \mathrm{C}$ during 30 min, then the mixture was used immediately. The starting alkylzinc-chlorides were obtained by transmetallation with $\mathrm{ZnCl}_{2}$ of the corresponding Grignard reagents, prepared in diethyl ether or THF by commercially available bromides or chlorides. The Grignard reagents from 3-chloro-1-propanol ${ }^{6}$ (entries 4 and 5 in Table 3) and 4-bromo-1-trimethylsilyl-1-butyne ${ }^{7}$ (entry 8 in Table 3,) were prepared in THF according to previously reported procedures. 4-Bromo-1-trimethylsilyl-1-butyne was sinthesized by a published procedure starting from 3-butyn-1-ol. ${ }^{8}(+)$-Dehydroabietylamine was a commercial product, purified by a published procedure. ${ }^{9}$ Commercial lithium chloride, lithium bromide, cuprous bromide, cuprous cyanide, heptakis(2,3,6-tri-O-methyl)- $\beta$-cyclodextrin, (R)-1-(1naphthyl)ethylamine and other chemicals were used as purchased.

\section{Spectroscopic and analytical data for all new acetylenic derivatives 3}

All compounds showed characteristic IR bands in the $3295-3310 \mathrm{~cm}^{-1}$ (三C-H stretching), $2100-2110 \mathrm{~cm}^{-1}\left(\mathrm{C} \equiv \mathrm{C}\right.$ stretching), and $630-645 \mathrm{~cm}^{-1}$ ( $\equiv \mathrm{C}-\mathrm{H}$ bending) regions.

3-[4-(2-Methylpropyl)phenyl]-1-butyne (3ad): 1H NMR $\delta 0.89(\mathrm{~d}, J=6.6 \mathrm{~Hz}, 6 \mathrm{H}), 1.50$ (d, $J=$ $7.0 \mathrm{~Hz}, 3 \mathrm{H}), 1.84(\mathrm{~m}, 1 \mathrm{H}), 2.25(\mathrm{~d}, J=2.5 \mathrm{~Hz}, 1 \mathrm{H}), 2.45(\mathrm{~d}, J=7.2 \mathrm{~Hz}, 2 \mathrm{H}), 3.74(\mathrm{dq}, J=2.5,7.0 \mathrm{~Hz}$, 1H), $7.12(\mathrm{~d}, J=8.0 \mathrm{~Hz}, 2 \mathrm{H}), 7.29(\mathrm{~d}, J=8.0 \mathrm{~Hz}, 2 \mathrm{H}) ;{ }^{13} \mathrm{C} \mathrm{NMR} \delta 22.2,24.1,30.1,31.1,44.9,69.9$ $(\equiv \mathrm{CH}), 87.4(-\mathrm{C} \equiv), 126.6,129.4,140.0,140.3 ; \mathrm{GC}-\mathrm{MS}(\mathrm{EI}) \mathrm{m} / \mathrm{z}$ (rel int) $186\left(\mathrm{M}^{+}, 47\right), 171(27), 143$ (100), 129 (77), 128 (64), 115 (16), 91 (14), 77 (8), 63 (6), 51 (5). Anal. Calcd. for $\mathrm{C}_{14} \mathrm{H}_{18}$ : C, 90.26; H, 9.74. Found: C, 90.30; H, 9.70. 
3-(2-Methoxyphenyl)-1-butyne (3ae): ${ }^{1} \mathrm{H}$ NMR $\delta 1.43(\mathrm{~d}, J=7.0 \mathrm{~Hz}, 3 \mathrm{H}), 2.19(\mathrm{~d}, J=2.4 \mathrm{~Hz}$, 1H), $3.79(\mathrm{~s}, 3 \mathrm{H}), 4.19(\mathrm{dq}, J=2.4,7.0 \mathrm{~Hz}, 1 \mathrm{H}), 6.80-7.60(\mathrm{~m}, 4 \mathrm{H}) ;{ }^{13} \mathrm{C}$ NMR $\delta 22.5,25.1,55.2,69.2$ ( $=\mathrm{CH}), 87.6(-\mathrm{C} \equiv), 110.4,120.8,127.7,127.9,131.1,156.2 ; \mathrm{GC}-\mathrm{MS}(\mathrm{EI}) \mathrm{m} / \mathrm{z}$ (rel int) $160\left(\mathrm{M}^{+}, 43\right)$, 145 (100), 127 (13), 115 (59), 102(17), 91 (36), 77 (17), 63 (14), 51 (14). Anal. Calcd. for $\mathrm{C}_{11} \mathrm{H}_{12} \mathrm{O}$ : C, 82.46; H, 7.55. Found: C, 82.48; H, 7.56.

3-(1-Naphthyl)-1-butyne (3ai): ${ }^{1} \mathrm{H}$ NMR $\delta 1.60(\mathrm{~d}, J=7.0 \mathrm{~Hz}, 3 \mathrm{H}), 2.28(\mathrm{~d}, J=2.5 \mathrm{~Hz}, 1 \mathrm{H}), 4.46$ $(\mathrm{dq}, J=2.5,7.0 \mathrm{~Hz}, 1 \mathrm{H}), 7.30-8.20(\mathrm{~m}, 7 \mathrm{H}) ;{ }^{13} \mathrm{C}$ NMR $\delta 22.9,28.0,70.4(\equiv \mathrm{CH}), 87.2(-\mathrm{C} \equiv), 123.0$, 124.2, 125.6, 125.7, 126.1, 127.7, 129.1, 130.5, 134.1, 138.3; GC-MS(EI) $m / z$ (rel int) $180\left(\mathrm{M}^{+}, 25\right)$, 179 (24), 165 (100), 89 (7), 83 (6). Anal. Calcd. for $\mathrm{C}_{14} \mathrm{H}_{12}$ : C, 93.29; H, 6.71. Found: C, 93.28; H, 6.72 .

3-(2-Naphthyl)-1-butyne (3aj): ${ }^{1} \mathrm{H}$ NMR $\delta 1.57$ (d, $\left.J=7.1 \mathrm{~Hz}, 3 \mathrm{H}\right), 2.32(\mathrm{~d}, J=2.5 \mathrm{~Hz}, 1 \mathrm{H}), 3.91$ $(\mathrm{dq}, J=2.5,7.1 \mathrm{~Hz}, 1 \mathrm{H}), 7.46(\mathrm{~m}, 3 \mathrm{H}), 7.80(\mathrm{~m}, 4 \mathrm{H}) ;{ }^{13} \mathrm{C} \mathrm{NMR} \delta 23.9,31.6,70.4(\equiv \mathrm{CH}), 87.0(-\mathrm{C} \equiv)$, 125.2, 125.5, 125.8, 126.2, 127.7, 127.9, 128.4, 132.6, 133.6, 140.1; GC-MS(EI) m/z (rel int) $180\left(\mathrm{M}^{+}\right.$, 50), 165 (100), 152 (4), 115 (7), 89 (10), 83 (7), 76 (7), 63 (5). Anal. Calcd. for $\mathrm{C}_{14} \mathrm{H}_{12}$ : C, 93.29; $\mathrm{H}$, 6.71. Found: C, 93.32; H, 6.68.

3-[(6-Methoxy)-2-naphthyl)]-1-butyne (3ak): ${ }^{1} \mathrm{H}$ NMR $\delta 1.58(\mathrm{~d}, J=7.1 \mathrm{~Hz}, 3 \mathrm{H}), 2.31$ (d, $J=2.4$ $\mathrm{Hz}, 1 \mathrm{H}), 3.90(\mathrm{dq}, J=2.4,7.1 \mathrm{~Hz}, 1 \mathrm{H}), 3.91(\mathrm{~s}, 3 \mathrm{H}), 7.12-7.76(\mathrm{~m}, 6 \mathrm{H}) ;{ }^{13} \mathrm{C} \mathrm{NMR} \delta 24.0,31.4,55.2$, $70.2(\equiv \mathrm{CH}), 87.2(-\mathrm{C} \equiv), 105.6,119.0,125.4,126.0,127.3,129.3,129.5,133.6,137.9,157.7$; GCMS(EI) $m / z$ (rel int) $210\left(\mathrm{M}^{+}, 64\right), 195$ (100), 165 (10), 152 (24). Anal. Calcd. for $\mathrm{C}_{15} \mathrm{H}_{14} \mathrm{O}: \mathrm{C}, 85.68$; H, 6.71. Found: C, 85.71; H, 6.73.

3-(2-Methoxyphenyl)-4,4-dimethyl-1-pentyne (3be): ${ }^{1} \mathrm{H}$ NMR $\delta 0.98(\mathrm{~s}, 9 \mathrm{H}), 2.11$ (d, $J=2.6 \mathrm{~Hz}$, 1H), $3.76(\mathrm{~s}, 3 \mathrm{H}), 4.15(\mathrm{~d}, J=2.6 \mathrm{~Hz}, 1 \mathrm{H}), 6.80-7.50(\mathrm{~m}, 4 \mathrm{H}) ;{ }^{13} \mathrm{C} \mathrm{NMR} \delta 27.1,35.4,39.8,55.0,69.7$ 
$(\equiv \mathrm{CH}), 86.5$ (-C $\equiv), 110.2,120.1,127.9,129.6,130.7,157.0 ; \mathrm{GC}-\mathrm{MS}(\mathrm{EI}) \mathrm{m} / z$ (rel int) $202\left(\mathrm{M}^{+}, 11\right)$, 187 (23), 172 (6), 146 (29), 145 (42), 131 (100), 115 (44), 102 (21), 91 (32), 76 (13), 57 (95). Anal. Calcd. for $\mathrm{C}_{14} \mathrm{H}_{18} \mathrm{O}$ : C, 83.12; H, 8.97. Found: C, 83.15; H, 9.00.

3-(4-Methoxyphenyl)-4,4-dimethyl-1-pentyne (3bf): ${ }^{1} \mathrm{H}$ NMR $\delta 0.96$ (s, 9H), 2.24 (d, $J=2.6 \mathrm{~Hz}$, $1 \mathrm{H}), 3.37(\mathrm{~d}, J=2.6 \mathrm{~Hz}, 1 \mathrm{H}), 3.79(\mathrm{~s}, 3 \mathrm{H}), 6.83(\mathrm{~m}, 2 \mathrm{H}), 7.23(\mathrm{~m}, 2 \mathrm{H}) ;{ }^{13} \mathrm{C}$ NMR $\delta 27.3,34.7,48.6$, 55.1, $71.2(\equiv \mathrm{CH}), 85.7(-\mathrm{C} \equiv), 113.1,130.6,134.8,158.7$; GC-MS(EI) $m / z$ (rel int) $202\left(\mathrm{M}^{+}, 11\right), 187$ (5), 146 (49), 145 (100), 131 (7), 102 (9), 57 (28). Anal. Calcd. for $\mathrm{C}_{14} \mathrm{H}_{18} \mathrm{O}: \mathrm{C}, 83.12 ; \mathrm{H}, 8.97$. Found: C, 83.17; H, 8.99.

3-(4-Dimethylaminophenyl)-4,4-dimethyl-1-pentyne (3bh): ${ }^{1} \mathrm{H}$ NMR $\delta 0.96(\mathrm{~s}, 9 \mathrm{H}), 2.21$ (d, $J=$ $2.6 \mathrm{~Hz}, 1 \mathrm{H}), 2.93(\mathrm{~s}, 6 \mathrm{H}), 3.33(\mathrm{~d}, J=2.6 \mathrm{~Hz}, 1 \mathrm{H}), 6.73(\mathrm{~m}, 2 \mathrm{H}), 7.24(\mathrm{~m}, 2 \mathrm{H}) ; \mathrm{GC}-\mathrm{MS}(\mathrm{EI}) \mathrm{m} / z$ (rel. int.) $215\left(\mathrm{M}^{+}, 8\right), 158(100), 142(10), 115$ (7). Anal. Calcd. for $\mathrm{C}_{15} \mathrm{H}_{21} \mathrm{~N}$ : C, 83.67; H, 9.83; N, 6.50. Found: C, 83.65; H, 9.82; N, 6.53 .

3-(1-Naphthyl)-4,4-dimethyl-1-pentyne (3bi): ${ }^{1} \mathrm{H}$ NMR $\delta 1.03(\mathrm{~s}, 9 \mathrm{H}), 2.22(\mathrm{~d}, J=2.5 \mathrm{~Hz}, 1 \mathrm{H})$, $4.48(\mathrm{~d}, J=2.5 \mathrm{~Hz}, 1 \mathrm{H}), 7.40-8.30(\mathrm{~m}, 7 \mathrm{H}) ;{ }^{13} \mathrm{C}$ NMR $\delta 27.9,36.4,42.6,70.6(\equiv \mathrm{CH}), 86.7(-\mathrm{C} \equiv)$, 124.0, 125.3, 125.5, 126.2, 127.9, 128.1, 129.3, 131.7, 132.5, 134.0; GC-MS(EI) m/z (rel int) 222 $\left(\mathrm{M}^{+}\right), 207$ (25), 165 (79), 164 (32), 163 (55), 141 (21), 139 (14), 126 (13), 67 (32), 57 (71), 41 (100). Anal. Calcd. for $\mathrm{C}_{17} \mathrm{H}_{18}$ : C, 91.84; H, 8.16. Found: C, 91.88; H, 8.12.

3-(2-Naphthyl)-4,4-dimethyl-1-pentyne (3bj): ${ }^{1} \mathrm{H}$ NMR $\delta 1.03$ (s, 9H), $2.30(\mathrm{~d}, J=2.4 \mathrm{~Hz}, 1 \mathrm{H})$, $3.59(\mathrm{~d}, J=2.4 \mathrm{~Hz}, 1 \mathrm{H}), 7.40-7.90(\mathrm{~m}, 7 \mathrm{H}) ;{ }^{13} \mathrm{C}$ NMR $\delta 27.5,35.1,46.9,71.6(\equiv \mathrm{CH}), 85.4(-\mathrm{C} \equiv)$, 125.8, 126.0, 127.2, 127.7, 128.0, 128.1, 128.4, 132.6, 133.1, 136.6; GC-MS(EI) m/z (rel int) $222\left(\mathrm{M}^{+}\right.$, 7), 207 (12), 165 (100), 152 (8), 128 (10), 57 (45), 41 (30). Anal. Calcd. for $\mathrm{C}_{17} \mathrm{H}_{18}$ : C, 91.84; H, 8.16. Found: C, 91.91; H, 8.09. 
3-(2-Methoxyphenyl)-3-methyl-1-pentyne (3ce): ${ }^{1} \mathrm{H}$ NMR $\delta 0.89(\mathrm{t}, J=7.0 \mathrm{~Hz}, 3 \mathrm{H}), 1.72(\mathrm{~s}, 3 \mathrm{H})$, $1.92(\mathrm{q}, J=7.0 \mathrm{~Hz}, 2 \mathrm{H}), 2.33(\mathrm{~s}, 1 \mathrm{H}), 3.73(\mathrm{~s}, 3 \mathrm{H}), 6.80-7.40(\mathrm{~m}, 4 \mathrm{H})$;GC-MS(EI) $\mathrm{m} / z$ (rel int) 188 (M+11), 159 (73), 144 (100), 128 (14), 115 (45), 103(6), 91 (8), 77 (19), 63 (16), 51 (22). Anal. Calcd. for $\mathrm{C}_{13} \mathrm{H}_{16} \mathrm{O}$ : C, 82.94; H, 8.57. Found: C, 83.00; H, 8.60.

3-(4-Methoxyphenyl)-3-methyl-1-pentyne (3cf): ${ }^{1} \mathrm{H}$ NMR $\delta 0.86(\mathrm{t}, J=7.3 \mathrm{~Hz}, 3 \mathrm{H}), 1.55$ (s, 3H), $1.79(\mathrm{q}, J=7.3 \mathrm{~Hz}, 2 \mathrm{H}), 2.36(\mathrm{~s}, 1 \mathrm{H}), 3.76(\mathrm{~s}, 3 \mathrm{H}), 6.85(\mathrm{~m}, 2 \mathrm{H}), 7.43(\mathrm{~m}, 2 \mathrm{H}) ;{ }^{13} \mathrm{C}$ NMR $\delta 9.5,29.6$, 36.8, 40.1, 55.0, $71.1(\equiv \mathrm{CH}), 89.6$ (-C $\equiv), 113.5,127.2,137.1,158.3$; GC-MS(EI) $m / z$ (rel int) 188 $\left(\mathrm{M}^{+}, 13\right), 159$ (100), 115 (10). Anal. Calcd. for $\mathrm{C}_{13} \mathrm{H}_{16} \mathrm{O}: \mathrm{C}, 82.94 ; \mathrm{H}$, 8.57. Found: C, 83.01; H, 8.60.

3-(1-Naphthyl)-3-methyl-1-pentyne (3ci): ${ }^{1} \mathrm{H}$ NMR $\delta 0.96$ (t, $\left.J=7.4 \mathrm{~Hz}, 3 \mathrm{H}\right), 1.83$ (s, 3H), 1.94 (m, 2H), $2.40(\mathrm{~s}, 1 \mathrm{H}), 7.36-8.30(\mathrm{~m}, 7 \mathrm{H}),{ }^{13} \mathrm{C}$ NMR $\delta 9.4,28.0,34.7,39.3,71.5(\equiv \mathrm{CH}), 90.6(-\mathrm{C} \equiv)$, 123.9, 125.0, 125.2, 125.6, 126.5, 128.4, 129.4, 131.2, 134.8, 135.0; GC-MS(EI) m/z (rel int) 208 (M', 14), 193 (17), 179 (100), 178 (29), 165 (7), $152(9)$.

3-(2-Naphthyl)-3-methyl-1-pentyne (3cj): ${ }^{1} \mathrm{H}$ NMR $\delta 0.88$ (t, $\left.J=7.4 \mathrm{~Hz}, 3 \mathrm{H}\right), 1.65$ (s, 3H), 1.91 $(\mathrm{q}, J=7.4 \mathrm{~Hz}, 2 \mathrm{H}), 2.45(\mathrm{~s}, 1 \mathrm{H}), 7.37-8.03(\mathrm{~m}, 7 \mathrm{H}) ;{ }^{13} \mathrm{C} \mathrm{NMR} \delta$ 9.6, 29.6, 36.4, 41.0, $71.7(\equiv \mathrm{CH})$, $89.3(-\mathrm{C} \equiv), 124.4,125.0,125.7,126.1,127.5,127.9,128.2,132.3,133.4,142.2 ;$ GC-MS(EI) $m / z($ rel int) $208\left(\mathrm{M}^{+}, 9\right), 179$ (100), 165 (6), 152 (12), 128 (7), 51 (7). Anal. Calcd. for $\mathrm{C}_{16} \mathrm{H}_{16}$ : C, 92.26; H, 7.74. Found: C, 92.30; H, 7.70.

3-(2-Ethylphenyl)-3,4,4-trimethyl-1-pentyne (3db): ${ }^{1} \mathrm{H}$ NMR $\delta 0.92$ (s, 9H), 1.17 (t, $J=7.5 \mathrm{~Hz}$, 3H), 1.69 (s, 3H), 2.27 (s, 1H), 2.57 (q, $J=7.5,2 \mathrm{H}), 7.10(\mathrm{~m}, 4 \mathrm{H})$. GC-MS(EI) $m / z$ (rel int) $214\left(\mathrm{M}^{+}\right)$, 199 (19), 164 (22), 157 (55), 143 (60), 142 (42), 129 (15), 57 (100), 41 (38). Anal. Calcd. for $\mathrm{C}_{16} \mathrm{H}_{22}$ : C, 89.65; H, 10.35. Found: C, 89.59; H, 10.41. 
3-(4-Ethylphenyl)-3,4,4-trimethyl-1-pentyne (3dc): ${ }^{1} \mathrm{H}$ NMR $\delta 0.89$ (s, 9H), 1.15 (t, $J=7.6 \mathrm{~Hz}$, $3 \mathrm{H}), 1.56(\mathrm{~s}, 3 \mathrm{H}), 2.24(\mathrm{~s}, 1 \mathrm{H}), 2.55(\mathrm{q}, J=7.6 \mathrm{~Hz}, 2 \mathrm{H}), 7.03(\mathrm{~m}, 2 \mathrm{H}), 7.36(\mathrm{~m}, 2 \mathrm{H}) ;{ }^{13} \mathrm{C} \mathrm{NMR} \delta 15.4$, 23.4, 26.3, 28.3, 36.9, 47.0, 70.9 ( $\equiv \mathrm{CH}), 90.3(-\mathrm{C} \equiv), 126.4,128.5,139.7,142.1$. GC-MS(EI) $\mathrm{m} / z$ (rel int) $214(\mathrm{M}+, 10), 199$ (30), 184 (15), 158 (62), 157 (84), 143 (41), 142 (27), 129 (19), 128 (7), 57 (100), 41 (36). Anal. Calcd. for $\mathrm{C}_{16} \mathrm{H}_{22}$ : C, 89.65; H, 10.35. Found: C, 89.66; H, 10.34.

3-(2-Methoxyphenyl)-3,4,4-trimethyl-1-pentyne (3de): ${ }^{1} \mathrm{H}$ NMR $\delta 0.99$ (s, 9H), 1.83 (s, 3H), 2.35 $(\mathrm{s}, 1 \mathrm{H}), 3.72(\mathrm{~s}, 3 \mathrm{H}), 6.80-7.40(\mathrm{~m}, 4 \mathrm{H}) ;{ }^{13} \mathrm{C}$ NMR $\delta 24.4,26.4,38.4,48.2,55.0,71.0(\equiv \mathrm{CH}), 91.6(-$ $\mathrm{C} \equiv), 112.4,119.7,128.1,130.0,133.2,158.7 ; \mathrm{GC}-\mathrm{MS}(\mathrm{EI}) \mathrm{m} / z$ (rel int) $201\left(\mathrm{M}^{+}-15,16\right), 186(10), 159$ (44), 145 (100), 144 (89), 128 (10), 115 (54), 91 (22), 77 (11), 65 (12), 57 (42), 41 (46). Anal. Calcd. for $\mathrm{C}_{15} \mathrm{H}_{20} \mathrm{O}: \mathrm{C}, 83.28 ; \mathrm{H}, 9.32$. Found: C, 83.31; H, 9.32.

3-(4-Methoxyphenyl)-3,4,4-trimethyl-1-pentyne (3df): ${ }^{1} \mathrm{H}$ NMR $\delta 0.95$ (s, 9H), 1.63 (s, 3H), 2.32 (s, 1H), $3.78(\mathrm{~s}, 3 \mathrm{H}), 6.75-7.60(\mathrm{~m}, 4 \mathrm{H}) ;{ }^{13} \mathrm{C} \mathrm{NMR} \delta 23.3,26.0,36.9,46.5,55.0,70.9$ (三CH), $90.3(-$ $\mathrm{C} \equiv), 112.4,129.7,134.8,158.2 ;$ GC-MS(EI) m/z (rel int) $216\left(\mathrm{M}^{+}, 9\right), 201$ (6), 187 (5), 160 (65), 159 (100), 145 (16), 128 (10), 115 (30), 89 (5), 77 (6), 57 (19), 41 (17). Anal. Calcd. for $\mathrm{C}_{15} \mathrm{H}_{20} \mathrm{O}: \mathrm{C}$, 83.28; H, 9.32. Found: C, 83.40; H, 9.34.

3-(4-Fluorophenyl)-3,4,4-trimethyl-1-pentyne (3dg): ${ }^{1} \mathrm{H}$ NMR $\delta 0.96$ (s, 9H), 1.64 (s, 3H), 2.35 (s, 1H), $6.97(\mathrm{~m}, 2 \mathrm{H}), 7.50(\mathrm{~m}, 2 \mathrm{H}) ;{ }^{13} \mathrm{C} \mathrm{NMR} \delta 23.3,26.0,36.9,46.8,71.3(\equiv \mathrm{CH}), 89.9(-\mathrm{C} \equiv), 113.8$ $\left(\mathrm{d}, J_{\mathrm{CF}}=21 \mathrm{~Hz}\right), 130.3\left(\mathrm{~d}, J_{\mathrm{CF}}=7.7 \mathrm{~Hz}\right), 138.4\left(\mathrm{~d}, J_{\mathrm{CF}}=3.4 \mathrm{~Hz}\right), 161.7\left(\mathrm{~d}, J_{\mathrm{CF}}=245 \mathrm{~Hz}\right) ; \mathrm{GC}-\mathrm{MS}(\mathrm{EI})$ m/z (rel int) $204\left(\mathrm{M}^{+}, 4\right), 201$ (6), 189 (10), 174 (6), 161 (7), 148 (62), 147 (50), 146 (24), 133 (21), 109 (6), 75 (6), 57 (100), 41 (33). Anal. Calcd. for $\mathrm{C}_{14} \mathrm{H}_{17} \mathrm{~F}: \mathrm{C}, 82.31$; H, 8.39. Found: C, 82.27; H, 8.40 . 
3-(4-Dimethylaminophenyl)-3,4,4-trimethyl-1-pentyne (3dh): ${ }^{1} \mathrm{H}$ NMR $\delta 0.96$ (s, 9H), 1.62 (s, 3H), $2.30(\mathrm{~s}, 1 \mathrm{H}), 2.93(\mathrm{~s}, 6 \mathrm{H}), 6.74(\mathrm{~m}, 2 \mathrm{H}), 7.24(\mathrm{~m}, 2 \mathrm{H})$; GC-MS(EI) $\mathrm{m} / z$ (rel int) $229\left(\mathrm{M}^{+}, 4\right), 214$ (1), 199 (1), 172 (100), 156 (4), 128 (4). Anal. Calcd. for $\mathrm{C}_{16} \mathrm{H}_{23} \mathrm{~N}$ : C, 83.79; H, 10.11; N, 6.11. Found: C, 83.70; H, 10.09; N, 6.21.

3-(2-Naphthyl)-3,4,4-trimethyl-1-pentyne (3dj): ${ }^{1} \mathrm{H}$ NMR $\delta 1.01$ (s, 9H), 1.76 (s, 3H), 2.41 (s, 1H), 7.40-7.90 (m, 7H); GC-MS(EI) m/z (rel int) $236\left(\mathrm{M}^{+}, 9\right), 180$ (66), 179 (100), 178 (40), 165 (24), 152 (9), 128 (10), 115 (6), 57 (41), 41 (24). Anal. Calcd. for $\mathrm{C}_{18} \mathrm{H}_{20}$ : C, 91.47; H, 8.53. Found: C, $91.45 ; \mathrm{H}, 8.55$.

3-[(6-Methoxy)-2-naphthyl)]-3,4,4-trimethyl-1-pentyne (3dk): ${ }^{1} \mathrm{H}$ NMR $\delta 0.93$ (s, 9H), 1.67 (s, 3H), 2.33 (s, 1H), 3.89 (s, 3H), 7.03-7.82 (m, 6H); GC-MS(EI) m/z (rel int) $266\left(\mathrm{M}^{+}, 8\right) 209$ (100), 165 (13), 57 (8). Anal. Calcd. for $\mathrm{C}_{19} \mathrm{H}_{22} \mathrm{O}$ : C, 85.67; H, 8.32. Found: C, 85.70; H, 8.35.

Spectroscopic and analytical data for all new allenic derivatives 4:

All compounds showed a characteristic IR band in the $1945-1955 \mathrm{~cm}^{-1}$ region $(\mathrm{C}=\mathrm{C}=\mathrm{C}$ stretching).

1-[4-(2-Methylpropyl)phenyl]-1,2-butadiene (4ad): GC-MS(EI) $m / z$ (rel int) $186\left(\mathbf{M}^{+}, 39\right)$, 143 (100), 128 (55), 115 (13), 91 (6), 77 (4), 51 (4).

1-(2-Methoxyphenyl)-1,2-butadiene (4ae): ${ }^{1} \mathrm{H}$ NMR $\delta 1.76(\mathrm{dd}, J=3.3,7.0 \mathrm{~Hz}, 3 \mathrm{H}), 3.77$ (s, 3H), $5.49(\mathrm{~m}, 1 \mathrm{H}), 6.50(\mathrm{dq}, J=3.3,7.0 \mathrm{~Hz}, 1 \mathrm{H}), 6.80-7.60(\mathrm{~m}, 4 \mathrm{H})$; GC-MS(EI) $\mathrm{m} / \mathrm{z}$ (rel int) 160 $\left(\mathrm{M}^{+}, 25\right), 145$ (100), 128 (13), 117 (34), 115 (71), 102(24), 91 (37), 75 (12), 63 (20), 51 (18). 
1-(1-Naphthyl)-1,2-butadiene (4ai): ${ }^{1} \mathrm{H}$ NMR $\delta 1.78(\mathrm{dd}, J=3.2,7.1 \mathrm{~Hz}, 3 \mathrm{H}), 5.52(\mathrm{~m}, 1 \mathrm{H})$, $6.76(\mathrm{dq}, J=3.2,7.1 \mathrm{~Hz}, 1 \mathrm{H}), 7.30-8.20(\mathrm{~m}, 7 \mathrm{H}) ;{ }^{13} \mathrm{C}$ NMR $\delta 13.9,88.5(\underline{\mathrm{CH}}=\mathrm{C}=), 90.6(=\mathrm{C}=\underline{\mathrm{CH}})$, $123.7,125.4,125.7,126.0,127.4,128.7,131.0,131.4,134.0,208.0$ (=C=); GC-MS(EI) $m / z$ (rel int) $180\left(\mathrm{M}^{+}, 49\right), 179$ (66), 165 (100), 152 (16), 89 (15), 83 (6), 76 (11), 63 (6). Anal. Calcd. for $\mathrm{C}_{14} \mathrm{H}_{12}$ : C, 93.29; H, 6.71. Found: C, 93.09; H, 6.89.

1-(2-Naphthyl)-1,2-butadiene (4aj): ${ }^{1} \mathrm{H}$ NMR $\delta 1.84(\mathrm{dd}, J=3.2,7.2 \mathrm{~Hz}, 3 \mathrm{H}), 5.61(\mathrm{~m}, 1 \mathrm{H})$, $6.27(\mathrm{dq}, J=3.2,7.0 \mathrm{~Hz}, 1 \mathrm{H}), 7.35-7.85(\mathrm{~m}, 7 \mathrm{H})$; GC-MS(EI) $\mathrm{m} / z$ (rel int) $180\left(\mathrm{M}^{+}, 81\right), 165(100)$, 152 (10), 139 (5) 115 (7), 89 (15), 83 (5), 76 (12), 63 (5), 51 (4).

1-[(6-Methoxy)-2-naphthyl)]-1,2-butadiene (4ak): ${ }^{1} \mathrm{H}$ NMR $\delta 1.82(\mathrm{dd}, J=3.2,7.0 \mathrm{~Hz}, 3 \mathrm{H})$, $3.91(\mathrm{~s}, 3 \mathrm{H}), 5.60(\mathrm{~m}, 1 \mathrm{H}), 6.24(\mathrm{dq}, J=3.2,7.0 \mathrm{~Hz}, 1 \mathrm{H}), 7.10-7.80(\mathrm{~m}, 6 \mathrm{H}) ; \mathrm{MS}(\mathrm{EI}) \mathrm{m} / z$ (rel int) 210 $\left(\mathrm{M}^{+}, 70\right), 195$ (100), $165(35), 152(8), 139(3), 89(10)$.

1-(2-Methoxyphenyl)-4,4-dimethyl-1,2-pentadiene (4be): ${ }^{1} \mathrm{H}$ NMR $\delta 1.12$ (s, 9H), 3.81 (s, $3 \mathrm{H}), 5.53(\mathrm{~d}, J=6.4 \mathrm{~Hz}, 1 \mathrm{H}), 6.60(\mathrm{~d}, J=6.4 \mathrm{~Hz}, 1 \mathrm{H}), 6.80-7.50(\mathrm{~m}, 4 \mathrm{H}) ;{ }^{13} \mathrm{C} \mathrm{NMR} \delta 30.2,32.5$, 55.4, $90.0(\underline{\mathrm{CH}}=\mathrm{C}=), 106.2(=\mathrm{C}=\underline{\mathrm{CH}}), 111.0,120.8,123.4,127.7,130.7,156.1,203.1(=\mathrm{C}=)$; GCMS(EI) m/z (rel int) $202\left(\mathrm{M}^{+}, 40\right), 187$ (39), 172 (9), 159 (7), 145 (34), 131 (100), 115 (58), 102 (32), 91 (30), 77 (19), 57 (83). Anal. Calcd. for $\mathrm{C}_{14} \mathrm{H}_{18} \mathrm{O}$ : C, 83.12; H, 8.97. Found: C, 82.98; H, 8.95.

1-(4-Methoxyphenyl)-4,4-dimethyl-1,2-pentadiene (4bf): ${ }^{1} \mathrm{H}$ NMR $\delta 1.11$ (s, 9H), 3.80 (s, $3 \mathrm{H}), 5.54(\mathrm{~d}, J=6.3 \mathrm{~Hz}, 1 \mathrm{H}), 6.15(\mathrm{~d}, J=6.3 \mathrm{~Hz}, 1 \mathrm{H}), 6.80-7.30(\mathrm{~m}, 4 \mathrm{H}) ;{ }^{13} \mathrm{C} \mathrm{NMR} \delta 30.1,32.4$, $55.2,95.6(\underline{\mathrm{CH}}=\mathrm{C}=), 106.9(=\mathrm{C}=\underline{\mathrm{CH}}), 114.2,127.6,131.1,158.7,202.1(=\mathrm{C}=) ; \mathrm{GC}-\mathrm{MS}(\mathrm{EI}) \mathrm{m} / z(\mathrm{rel}$ int) $202\left(\mathrm{M}^{+}, 43\right), 187(12), 145$ (100), $131(6), 115(6), 102(8), 77$ (6), 57 (39). 
1-(4-Dimethylaminophenyl)-4,4-dimethyl-1,2-pentadiene (4bh): ${ }^{1} \mathrm{H}$ NMR $\delta 1.11(\mathrm{~s}, 9 \mathrm{H})$, $2.93(\mathrm{~s}, 6 \mathrm{H}), 5.53(\mathrm{~d}, J=6.4 \mathrm{~Hz}, 1 \mathrm{H}), 6.60(\mathrm{~d}, J=6.4 \mathrm{~Hz}, 1 \mathrm{H}), 6.69(\mathrm{~m}, 2 \mathrm{H}), 7.18(\mathrm{~m}, 2 \mathrm{H})$; GCMS(EI) $m / z$ (rel int) $215\left(\mathrm{M}^{+}, 30\right), 200(7), 158$ (100), 142 (9), 115 (8).

1-(1-Naphthyl)-4,4-dimethyl-1,2-pentadiene (4bi): ${ }^{1} \mathrm{H}$ NMR $\delta 1.16$ (s, 9H), 5.62 (d, $J=6.4$ $\mathrm{Hz}, 1 \mathrm{H}), 6.91(\mathrm{~d}, J=6.4 \mathrm{~Hz}, 1 \mathrm{H}), 7.44-8.32(\mathrm{~m}, 7 \mathrm{H}) ;{ }^{13} \mathrm{C}$ NMR $\delta 30.3,32.8,92.9(\mathrm{CH}=\mathrm{C}=), 106.2$ $(=\mathrm{C}=\underline{\mathrm{CH}}), 123.8,125.0,125.9,126.0(2 \mathrm{C}), 127.5,128.9,131.1,134.3,136.0,204.5$ (=C=); GCMS(EI) m/z (rel int) $222\left(\mathrm{M}^{+}, 16\right), 207$ (45), 192 (10), 179 (31), 165 (100), 164 (27), 163 (35), 152 (24), 139 (14), 115 (14), 89 (8), 75 (8), 57 (50), 41 (45). Anal. Calcd. for $\mathrm{C}_{17} \mathrm{H}_{18}$ : C, 91.84; H, 8.16. Found: C, 91.73; H, 8.21.

1-(2-Naphthyl)-4,4-dimethyl-1,2-pentadiene (4bj): ${ }^{1} \mathrm{H}$ NMR $\delta 1.16$ (s, 9H), 5.64 (d, $J=6.3$ $\mathrm{Hz}, 1 \mathrm{H}), 6.37(\mathrm{~d}, J=6.3 \mathrm{~Hz}, 1 \mathrm{H}), 7.40-7.90(\mathrm{~m}, 7 \mathrm{H}) ;{ }^{13} \mathrm{C} \mathrm{NMR} \delta 96.6(\underline{\mathrm{CH}}=\mathrm{C}=), 107.2(=\mathrm{C}=\underline{\mathrm{CH}})$, 203.5 (=C=); GC-MS(EI) m/z (rel int) $222\left(\mathrm{M}^{+}, 26\right), 207$ (47), 192 (12), 179 (30), 165 (100), 164 (40), 152 (20), 139 (10), 89 (6), 57 (52).

1-(2-Methoxyphenyl)-3-methyl-1,2-pentadiene (4ce): ${ }^{1} \mathrm{H}$ NMR $\delta 1.05$ (t, $\left.J=7.4 \mathrm{~Hz}, 3 \mathrm{H}\right)$, $1.79(\mathrm{~d}, J=3.0 \mathrm{~Hz}, 3 \mathrm{H}), 2.07(\mathrm{dq}, J=3.0,7.4 \mathrm{~Hz}, 2 \mathrm{H}), 3.78(\mathrm{~s}, 3 \mathrm{H}), 6.50(\mathrm{~m}, 1 \mathrm{H}), 6.70-7.50(\mathrm{~m}, 4 \mathrm{H})$; ${ }^{13} \mathrm{C}$ NMR $\delta 12.1,18.5,26.9,55.4,88.1(\underline{\mathrm{C}}=\mathrm{C}=), 104.6(=\mathrm{C}=\underline{\mathrm{CH}}), 110.9,120.7,124.5,127.5(2 \mathrm{C})$, 156.1, 202.9 (=C=); GC-MS(EI) m/z (rel int) $188\left(\mathrm{M}^{+}, 26\right), 173$ (100), 159 (40), 158 (34), 144(20), 131 (39), 115 (56), 102 (9), 91 (45), 89 (22), 77 (25), 63 (23). Anal. Calcd. for $\mathrm{C}_{13} \mathrm{H}_{16} \mathrm{O}: \mathrm{C}, 82.94$; H, 8.57. Found: C, 82.86; H, 8.60.

1-(4-Methoxyphenyl)-3-methyl-1,2-pentadiene (4cf): ${ }^{1} \mathrm{H}$ NMR $\delta 1.05$ (t, $\left.J=7.3 \mathrm{~Hz}, 3 \mathrm{H}\right)$, $1.79(\mathrm{~d}, J=3.0 \mathrm{~Hz}, 3 \mathrm{H}), 2.07(\mathrm{dq}, J=3.0,7.3 \mathrm{~Hz}, 2 \mathrm{H}), 3.74(\mathrm{~s}, 3 \mathrm{H}), 6.05(\mathrm{~m}, 1 \mathrm{H}), 6.81(\mathrm{~m}, 2 \mathrm{H}), 7.19$ $(\mathrm{m}, 2 \mathrm{H}) ;{ }^{13} \mathrm{C}$ NMR $\delta 12.1,18.6,27.1,55.1,93.9(\underline{\mathrm{C}}=\mathrm{C}=), 105.3(=\mathrm{C}=\underline{\mathrm{CH}}), 114.1,127.5,128.6,158.6$, 
201.9 (=C=); GC-MS(EI) m/z (rel int) $188\left(\mathrm{M}^{+}, 31\right), 173$ (100), 159 (38), 158 (40), 144 (16), 115 (42), $91(18)$.

1-(1-Naphthyl)-3-methyl-1,2-pentadiene (4ci): ${ }^{1} \mathrm{H}$ NMR $\delta 1.10(\mathrm{t}, J=7.3 \mathrm{~Hz}, 3 \mathrm{H}), 1.86$ (d, $J$ $=3.0 \mathrm{~Hz}, 3 \mathrm{H}), 2.13(\mathrm{dq}, \mathrm{J}=3.0,7.3 \mathrm{~Hz}, 2 \mathrm{H}), 6.78(\mathrm{~m}, 1 \mathrm{H}), 7.37-8.28(\mathrm{~m}, 7 \mathrm{H}) ;{ }^{13} \mathrm{C} \mathrm{NMR} \delta 12.1,18.7$, $27.1,91.0(\underline{\mathrm{C}}=\mathrm{C}=), 104.2(=\mathrm{C}=\underline{\mathrm{CH}}), 123.8,125.1,125.6,125.7,125.9,127.1,128.7,131.0,132.4$, 134.1, 204.3 (=C=); GC-MS(EI) m/z (rel int) 208 (M+1 53), 193 (45), 179 (100), 178 (40), 165 (14), 152 (17), 89 (6). Anal. Calcd. for $\mathrm{C}_{16} \mathrm{H}_{16}$ : C, 92.26; H, 7.74. Found: C, 92.17; H, 7.83.

1-(2-Naphthyl)-3-methyl-1,2-pentadiene (4cj): ${ }^{1} \mathrm{H}$ NMR $\delta 1.08(\mathrm{t}, J=7.3 \mathrm{~Hz}, 3 \mathrm{H}), 1.85$ (d, $J$ $=3.0 \mathrm{~Hz}, 3 \mathrm{H}), 2.12(\mathrm{dq}, J=3.0,7.3 \mathrm{~Hz}, 2 \mathrm{H}), 6.26(\mathrm{~m}, 1 \mathrm{H}), 7.36-7.85(\mathrm{~m}, 7 \mathrm{H}) ;{ }^{13} \mathrm{C} \mathrm{NMR} \delta 12.1,18.7$, $27.1,94.9(\underline{\mathrm{C}}=\mathrm{C}=), 105.8(=\mathrm{C}=\underline{\mathrm{CH}}), 124.8,125.1,125.4,126.2,127.7,127.8,128.2,132.6,133.9$, 134.0, 203.3 (=C=); GC-MS(EI) m/z (rel int) $208\left(\mathrm{M}^{+}, 49\right), 193$ (46), 179 (100), 178 (93), 165 (21), 151 (29), 139 (11), 115 (14), 99 (6), 91 (6), 76 (16), 63 (13), 51 (11). Anal. Calcd. for $\mathrm{C}_{16} \mathrm{H}_{16}$ : $\mathrm{C}$, 92.26; H, 7.74. Found: C, 92.13; H, 7.85.

1-(2-Ethylphenyl)-3,4,4-trimethyl-1,2-pentadiene (4db): ${ }^{1} \mathrm{H}$ NMR $\delta 1.05$ (s, 9H), 1.16 (t, $J=$ $7.6 \mathrm{~Hz}, 3 \mathrm{H}), 1.73(\mathrm{~d}, J=2.9 \mathrm{~Hz}, 3 \mathrm{H}), 2.55(\mathrm{q}, \mathrm{J}=7.6 \mathrm{~Hz}, 2 \mathrm{H}), 6.20(\mathrm{q}, J=2.9,1 \mathrm{H}), 7.10(\mathrm{~m}, 4 \mathrm{H})$; GC-MS(EI) m/z (rel int) $214\left(\mathrm{M}^{+}, 25\right), 199$ (24), 184 (7), 171 (6), 157 (68) 143 (28), 142 (20), 129 (7), 57 (100). Anal. Calcd. for $\mathrm{C}_{16} \mathrm{H}_{22}$ : C, 89.65; H, 10.35. Found: C, 89.51; H, 10.47.

1-(4-Ethylphenyl)-3,4,4-trimethyl-1,2-pentadiene (4dc): ${ }^{1} \mathrm{H}$ NMR $\delta 1.05$ (s, 9H), $1.14(\mathrm{t}, J=$ $7.6 \mathrm{~Hz}, 3 \mathrm{H}), 1.71(\mathrm{~d}, J=2.9 \mathrm{~Hz}, 3 \mathrm{H}), 2.54(\mathrm{q}, J=7.6 \mathrm{~Hz}, 2 \mathrm{H}), 5.95(\mathrm{q}, J=2.9 \mathrm{~Hz}, 1 \mathrm{H}), 7.07(\mathrm{~m}, 4 \mathrm{H})$; ${ }^{13} \mathrm{C}$ NMR $\delta 14.7,15.7,28.6,29.2,34.2,93.9(\mathrm{C}=\mathrm{C}=), 112.4(=\mathrm{C}=\underline{\mathrm{CH}}), 126.3,128.1,133.6,142.4$, 201.5 (=C=); GC-MS(EI) m/z (rel int) 214 (M+, 32), 199 (35), 184 (12), 171 (4), 157 (72) 143 (30), $142(20), 129(11), 57(100)$. 
1-(4-Dimethylaminophenyl)-3,4,4-trimethyl-1,2-pentadiene (4dh): ${ }^{1} \mathrm{H}$ NMR $\delta 1.20(\mathrm{~s}, 9 \mathrm{H})$, $1.86(\mathrm{~d}, J=2.8 \mathrm{~Hz}, 3 \mathrm{H}), 2.98(\mathrm{~s}, 6 \mathrm{H}), 6.08(\mathrm{q}, J=2.8 \mathrm{~Hz}, 1 \mathrm{H}), 6.80(\mathrm{~m}, 2 \mathrm{H}), 7.27(\mathrm{~m}, 2 \mathrm{H})$; GCMS(EI) $m / z$ (rel int) $229\left(\mathrm{M}^{+}, 26\right), 214$ (18), 172 (100), 156 (7), 129(10).

1-[(6-Methoxy)-2-naphthyl)]-3,4,4-trimethyl-1,2-pentadiene (4dk): ${ }^{1} \mathrm{H}$ NMR $\delta 1.16$ (s, 9H), $1.85(\mathrm{~d}, J=2.8 \mathrm{~Hz}, 3 \mathrm{H}), 3.91(\mathrm{~s}, 3 \mathrm{H}), 6.20(\mathrm{q}, J=2.8 \mathrm{~Hz}, 1 \mathrm{H}), 7.09-7.69(\mathrm{~m}, 6 \mathrm{H}) ; \mathrm{GC}-\mathrm{MS}(\mathrm{EI}) \mathrm{m} / z$ (rel int) $266\left(\mathrm{M}^{+}, 26\right) 209$ (100), 165 (12), 57 (14), 41 (6).

\section{References}

(1) (a) Landor, S.R.; Patel, A.N.; Wither, P.F. J. Chem. Soc., C 1966, 1223-1226. (b) Montury, M.; Goré, J. Synth. Commun. 1980, 10, 873-879. (c) Elsevier, C.J.; Vermeer, P.; Gedanken, A.; Runge, W. J. Org. Chem. 1985, 50, 364-367. (d) Caporusso, A.M.; Zoppi, A.; Da Settimo, F.; Lardicci, L. Gazz. Chim. Ital. 1985, 115, 293-295.

(2) (a) Weidmann, R.; Schoofs, A.; Horeau, A. Bull. Soc. Chim. Fr. 1976, 645-648. (b) Olsson, L.; Claesson, A. Acta Chem. Scand. 1977, B31, 614-618. (c) Elsevier, J.C.; Mooiweer, H.H. J. Org. Chem. 1987, 52, 1536-1539. (d) Caporusso, A.M.; Rosini, C.; Lardicci, L.; Polizzi, C.; Salvadori, P. Gazz. Chim. Ital. 1986, 116, 467-469. (e) Uccello-Barretta, G.; Balzano, F.; Caporusso, A.M.; Iodice, A.; Salvadori, P. J. Org. Chem. 1995, 60, 2227-2231. (f) Hickmam, J.R.; Kenyon, J. J. Chem. Soc. 1955, 2051-2052. (g) Bertrand, M.; Gil, G.; Kumar, a. Nouv. J. Chim. 1980, 4, 69-72.

(3) Uccello-Barretta, G.; Balzano, F.; Caporusso, A.M.; Salvadori, P. J. Org. Chem. 1994, 59, 836-839.

(4) Lamneck Jr, J.H. J. Am. Chem. Soc. 1954, 76, 1106-1107. 
(5) Knochel, P.; Singer, R.D. Chem. Rev. 1993, 93, 2117-2188.

(6) Cahiez, G.; Alexakis, A.; Normant, J.F. Tetrahedron Lett. 1978, 19, 3013-3014.

(7) Rossi, R.; Carpita, A.; Ciofalo, M.; Lippolis, V. Tetrahedron 1991, 47, 8443-8460.

(8) Overman, L.E.; Brown, M.J.; McCann, S.F. Org. Synt. 1989, 68, 182.

(9) Gottstein, W.J.; Cheney, L.C. J. Org. Chem. 1965, 30, 2072-2073. 